\title{
BIM MIXED REALITY TOOL FOR THE INSPECTION OF HERITAGE BUILDINGS
}

\author{
C. Brito ${ }^{1}$, N. Alves ${ }^{1, *}$, L. Magalhães ${ }^{2}$, M. Guevara ${ }^{1}$ \\ ${ }^{1}$ Centro de Computação Gráfica,Campus de Azurém, Edifício 14, 4800-058, Guimarães - Portugal \\ \{cbrito, nelson.alves, miguel.guevara\}@ccg.pt \\ ${ }^{2}$ Centro ALGORITMI, Universidade do Minho,Campus de Azurém, 4800-058 Guimarães - Portugal \\ lmagalhaes@dsi.uminho.pt
}

Commission II, WG II/8

KEY WORDS: BIM, Mixed Reality, IFC, Heritage Conservation, Unity, Inspection Software, Preventive Maintenance

\begin{abstract}
:
Historical and cultural building conservation is often limited to high value buildings and only receives financial support when serious issues arise. Modern technologies are able to support and encourage a preventive approach, diminishing costs and avoiding possibly irreparable damage. One way of doing so is by improving the work-flow of building inspectors during their inspection routines. In that regard, mixed reality presents itself as an attractive framework for easing maintenance tasks. This paper describes our current implementation of such a solution, levering the spatial capabilities of Microsoft's HoloLens glasses.
\end{abstract}

\section{INTRODUCTION}

In this article we present our approach towards the development of a mixed reality application for assisting in the inspection of heritage buildings. We continue the work initiated in (Fonnet et al., 2017) and will discuss relevant aspects stemming from our original conception to the current state of implementation. This includes:

- How building information is commonly stored and what processing steps must be taken in order to use it effectively in a real-time application.

- How to interact with spatial information for overlaying of virtual models in real space.

- How to leverage existing frameworks to assist in development and how to design an intuitive and efficient user interface.

\section{BUILDING INFORMATION MODELLING}

BIM is currently the most common denomination for a new way of approaching the design, construction and maintenance of buildings. It has been defined as "a set of interacting policies, processes and technologies generating a methodology to manage the essential building design and project data in digital format throughout the building's life-cycle" (Succar, 2009). One of the most valuable functions of BIM is its ability to improve the coordination between multiple design disciplines, thus reducing errors.

Among the most commonly used data exchange standards for BIM is the Industry Foundation Classes (IFC) led by buildingSMART. This standard plays a crucial role to facilitate interoperability between various software platforms in the Architecture,

\footnotetext{
${ }^{*}$ Corresponding author
}

Engineering and Construction (AEC) industry. To date, the IFC standard has been widely supported by the market-leading BIM software vendors (Gao et al., 2015). In essence, IFC provides a global database for construction projects by linking all aspects of their construction. This includes the parametric geometry which constitutes the building itself, the constituting materials, as well as facilities, such as the heating and electrical subsystems. Leading architectural software suites, such as Autodesk Revit, allow for exporting and importing IFC data, and as such we opted to use IFC as input/output format for our platform.

In the context of historical buildings, geometry is not designed from scratch, but built from an assortment of real life measurements, such as laser scanning and digital imaging. This data usually presents erroneous measurements and artifacts, thus requiring processing and refinement until the final 3D geometry can be obtained (Murphy et al., 2009). Having this model is a powerful asset for conservation and preventive maintenance, and allows for further development of applications, such as the one presented here.

\section{PREVENTIVE MAINTENANCE}

Preventive conservation includes any strategies, actions, skills, and judgments to balance heritage protection and public access (Terminology, Resolution, 2008). Its plan for a publicly accessible buildings involves a variety of topics in order to assure damage minimization, users' comfort, energy efficiency, education, and safety precaution. It requires the achievement of the right equilibrium between a number of complex and frequently contradictory environmental parameters (Lucchi, 2018).

As a result of recent developments, technology-driven approaches have begun taking form, based on concepts such as IoT (Internet of Things), leveraging the use of sensor networks for constant monitoring of conditions (Mesas-Carrascosa et al., 2016) (Perles et al., 2018). However, in buildings there are generally very large areas to cover, as well as multitude of possible damages. As such, this sort of preventive maintenance requires periodic examinations conducted by trained professionals. 
Unfortunately, no systematic policy for the preventive conservation of built cultural heritage currently exists in the SouthWest Europe. The actual approaches for inspection, diagnosis, monitoring and curative conservation are intermittent, unplanned, overpriced and lack a methodical strategy. In response, we have established a comprehensive form containing a set of metrics and records, as well a database of damages which commonly arise with the passage of time, such as biological colonization and discoloration and deposits. Through periodical inspections and a centralized storage of the damages found it is possible to accompany their progression and take appropriate, timely action.

\section{MIXED REALITY}

Mixed Reality, also referred to as Hybrid reality, consists in combining real and digital worlds, to create immersive applications where both worlds interact. Mixed Reality can be considered a superset of Augmented Reality, with the distinguishing factors that virtual objects are anchored to the real world and the user may interact with them.

Mixed reality has had several successful applications in fields such as health-care (Hanna et al., 2018), education and training (Wu et al., 2013), architecture, engineering and construction (Chi et al., 2013) and industrial maintenance (Gavish et al., 2015), which suggests it could be of use in this scenario as well. We found Mixed Reality to be well suited to the scope of this project for two main reasons. First, it allows the inspector to replace an assortment of tools, such as a laptop/camera/microphone/clipboard, with a single device. It follows that the required forms can be filled more efficiently. Secondly, by visualizing the 3D architectural model it is possible to directly register any found defects or damages in their respective location. This allows for an objective and intuitive visualization of the damages found once the inspection is complete, as well as keeping track of their evolution and all measures taken to combat them.

\subsection{The HoloLens}

The HoloLens by Microsoft Corp. is the world's first untethered Mixed Reality (MR) Head Mounted Display (HMD) system, released to developers in March 2016 as a Development Kit. It possesses the unusual capability to perceive the space around it and thus maintaining a coordinate system which can be used to place objects, or holograms, in three dimensional space, directly in the user's field of view. This is made possible by an array of cameras and sensors. Features are continuously extracted from this data and processed to generate an approximate representation of the surrounding environment. This process is referred to as Spatial Mapping and produces a Spatial Mesh. Additionally, the HoloLens is equipped with a general purpose camera, which allows for video and photo capture, as well as a microphone for audio recording.

4.1.1 Limitations Striving to be completely portable, these devices unfortunately suffer multiple limitations, such as the lack of battery time (about 180 minutes with full charge), a narrow field of view (35), limited processing capabilities and lack of high quality depth data (Garon et al., 2016). The user interaction is also limited to two gestures, the air tap and the bloom, the later being reserved to exit an application. Fortunately, both hands are trackable by the device, which allows for widening the range of available gestures, such as approaching or parting both hands. The device also has a tendency to heat up when under heavy processing load, which makes it uncomfortable to wear for long periods of time. This is mostly due to the experimental nature of the HoloLens, given that it is the first of its kind, and will certainly be improved upon over the next device iterations.

4.1.2 Development Mixed reality apps are built with the Universal Windows Platform. The two main approaches available are using DirectX and the Unity engine. DirectX grants the most control but is very time consuming as it entails more domain-specific knowledge. The option recommended by Microsoft itself is the use of the Unity game engine. We chose this approach, which also allows us to make use of the Mixed Reality Toolkit (MRTK). This framework provides implementations for common problems that arise in mixed reality development, such as camera management, user input and interaction with the spatial mesh.

\section{THE PROPOSED SYSTEM}

Our goal is to create an application to be run on the HoloLens device for a simple and efficient registration of information pertaining to the conservation status of historical buildings. This application contains the following main features:

- Overlay the architectural model to the real world

- Fill the inspection form by selecting appropriate damage categories

- Assign damage reports to building locations

- Associate photographs and audio recordings to damages reports

\subsection{Architectural Model overlay}

5.1.1 Geometry Extraction As mentioned in section 2, we chose IFC as the input format for architectural data. This is greatly due the fact that it is an open interchange format, that it is widely supported by commercial products and that it satisfies the requirements of our application regarding storage of information. Due to its open nature, various open-source software libraries have been developed to process and manipulate these files, which also contributes to making it an attractive choice for development.

In IFC files, as with other CAD files (such as STEP), geometry is defined implicitly, by curves, surfaces and other parametric geometrical primitives. This representation is suited for representing objects without loss of detail, but it is not suitable for real-time rendering, as game engines (and graphics hardware) are specialized in rendering polygonal meshes. As such, we make use of the open-source IfcOpenShell application for converting the implicit geometry in IFC files into explicit geometry geometry data required by the Unity engine. IfcOpenShell is built atop of the OpenCascades framework.

We take care to export this 3D model in real scale, while preserving the IFC GUIDs (Global unique identifiers) of each submodel, so that annotations can be correctly associated with a building element. 
5.1.2 Model Overlay As mentioned in section 4.1, the HoloLens is able to map the space around it. The space is represented as a tessellated mesh, referred to as the Spatial Mesh. One of our objectives is to allow the software to place the 3D architectural model automatically, based on where the user is located within the building. This is an instance of a common problem in computer vision, by the name of point set registration. As the name implies, this is the process of finding a spatial transformation that aligns two point sets. In this instance, one point set is the building's 3D model, while the other is the spatial mapping produced by the HoloLens.

The most common method for tackling the registration problem is ICP, which stands for Iterative Closest Point, originally presented in (Besl, McKay, 1992). The basic version of this algorithm operates by assuming an initial matching between the point sets, which is then refined iteratively so as to minimize the distance between both point sets until a certain termination criteria is met. Since the original paper several variants of this algorithm have been proposed (Rusinkiewicz, Levoy, 2001), (Bouaziz et al., 2013).

As was suggested in (Fonnet et al., 2017), we attempted this approach but had little success. We found this to be due to the following reasons:

\section{ICP is a family of resource intensive algorithms This} makes them unsuitable for deployment in environments with limited hardware capabilities, such as the HoloLens, which results in unfeasible execution times.

Spatial mapping data is noisy The reconstruction of the space around the HoloLens is very coarse (Garon et al., 2016). This adds a further layer of difficulty to the problem, as the data would require further processing prior to matching attempts.

Spatial mapping is very local Only a small portion of the building depth data is available at one time matching. As the user moves around the building the spatial data does become more complete, but this is not a practical solution.

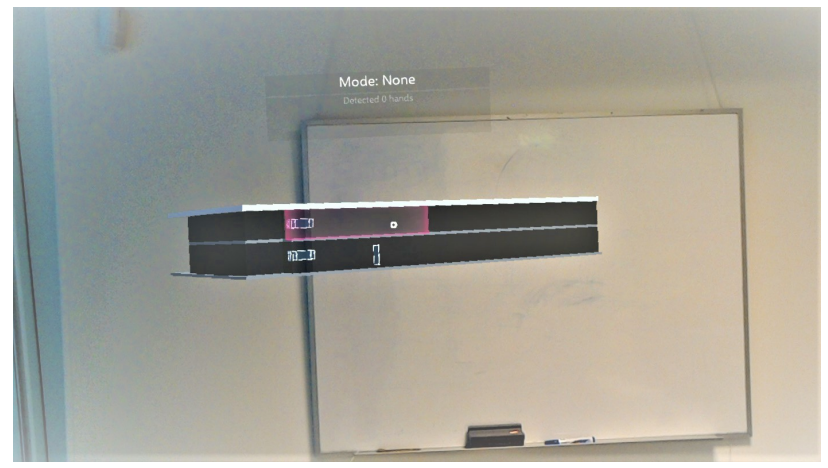

Figure 1. Showing the building miniature.

As an alternative, we implemented a manual approach for this registration. It consists in displaying a miniature of the building to the user (as can be seen on figure 1), and allowing them to select key-object which will then be placed on the spatial mapping mesh. This is accomplished by using the user gaze as a pointing device. The building is decomposed as the user zooms in, allowing them to select any desired key-object, as can be seen in figure 2 . The key object placement can be seen in figure

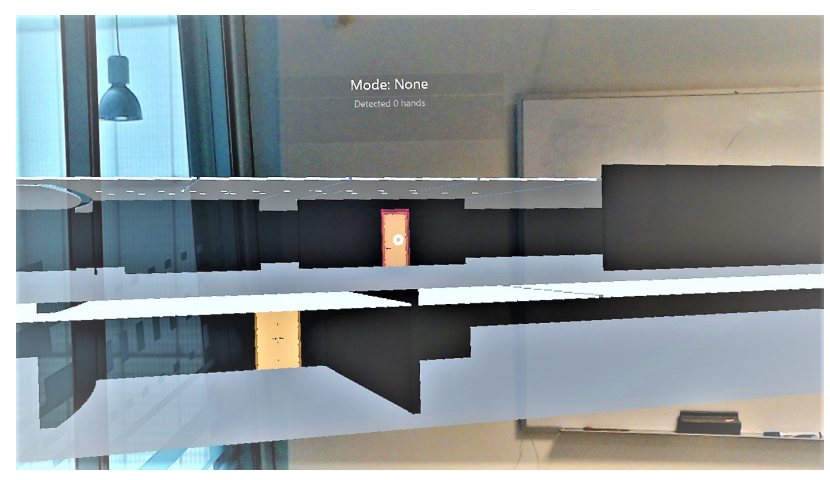

Figure 2. Zooming to the desired key-object.

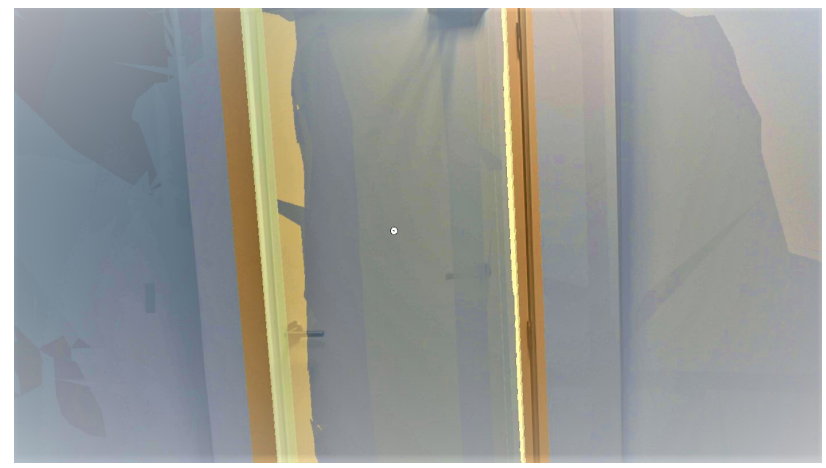

Figure 3. The door was selected as key-object.

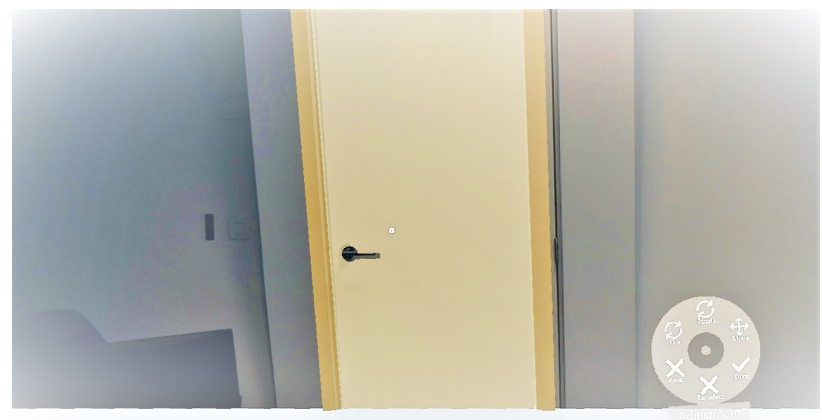

Figure 4. Matching the door's position/rotation to the real world.

3. Afterwards the position/orientation can be refined to align the object, shown on figure 4. Using this key-object's local position and rotation the whole building's position and rotation are then inferred. This key object can be any constituent part of the building, such as doors, walls or windows.

\subsection{Damage Report}

Our damage report consists in a set of damage entries chosen from a global atlas of possible damages. To these damages, the inspector may associate either photographs or audio recordings. Each damage is characterized by a class, a sub-class, and a subsub-class. For instance, Algae belongs to the Microorganisms sub-class, which belongs to the Biological Colonization class.

We assume that there will be no internet connectivity on the inspection site, and thus can not rely on internet-based services, such as speech-to-text conversion. This also influences how information is stored. As a result, we keep a local database of changes that the user has made. We have used JSON (JavaScript Object Notation) for this purpose, as it is simple, light- 
weight and may be sent to our central server directly for persistent storage once internet connectivity is restored.

\subsection{User Interaction}

One of the earliest challenges we faced was to develop an interaction system which allowed for fast traversal of application states, given the set of available options the software presents.

The HoloLens uses eye gaze direction as its primary pointing device, so typical user interfaces rely on the user looking at a particular UI (user interface) element and performing the Tap gesture to interact with it. In order to quicken this process, we developed a radial menu which is controlled solely by the tap gesture. The user simply taps and drags their hand towards the intended option, which will trigger the interaction as the tap is released. We found this approach to be successful since most users have found it intuitive to use.

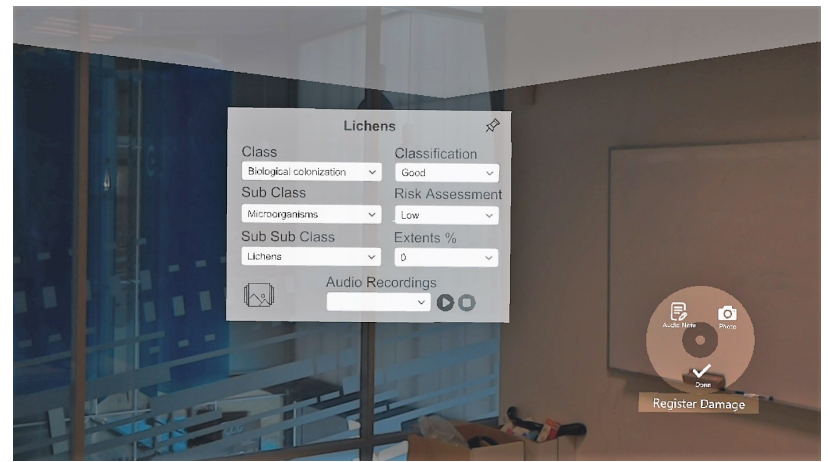

Figure 5. The main damage form.

Regarding the filling of the inspection form, a more traditional window-based user interface was designed which follows the user when active and allows damage class/sub-class/sub-subclass to be selected, as well as various severity indicators. This is shown in figure 5 .

\subsection{Damage annotations}

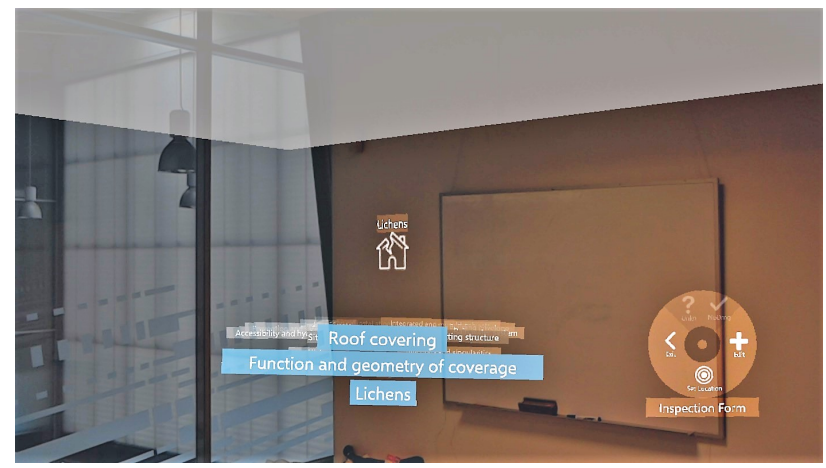

Figure 6. Annotating the damage Lichens to the wall.

The remaining key feature in our system is the ability to assign damages to building locations. In terms of user interface, as with key-object placement, this is also accomplished with gaze placement. As with the key object placement, this consists in placing the virtual annotation aligned with the user's gaze direction and upon movement the annotation is attached to the nearest surface (in this case, the building element the user is looking at). Upon confirmation, by tap gesture, the annotation's location is fixed. This process is shown in figure 6 .
We then determine the object upon which the user anchored the damage annotation and calculate its coordinates relative to it. As previously explained, when we export the 3D model we preserve the global identifiers from the IFC file exactly for this purpose. This information is then stored in the local JSON database.

The actual annotation process is made using the open-source $\mathrm{C \#}$ xBim library. Ideally this would be done within the HoloLens application. However, this had to be implemented in an external application, due to technical limitations of the C\# language support in the HoloLens. In this external application, the JSON database is parsed and the corresponding annotations are created and added to the original IFC file.

The process is specific to how IFC stores data and involves the addition of an IfcAnnotation element for each registered damage. These elements must each be associated with a geometric representation and a position, which is relative to the building element where the damages were found. This involves the use of multiple IFC classes as illustrated in figure 7. The IfcLocalPlacement class indicates that our annotation should be placed relatively to our chosen object, while the IfcProductDefinitionShape represents our selected geometry, in this case, a simple bounding box. The size and origin of this bounding box are then set with IfcPositiveLengthMeasure and IfcCartesianPoint respectively.

\section{IfcAnnotation}

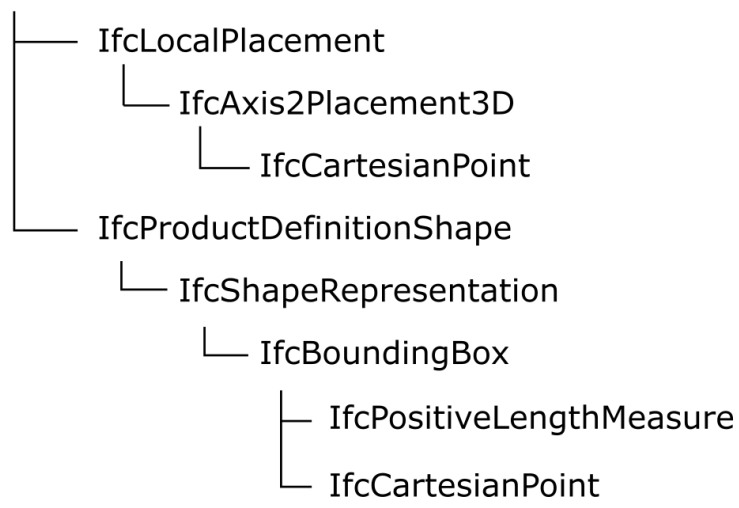

Figure 7. Our IFC annotation structure.

As a result, this damage annotation can then be visualised in specialized software, such as the open-source xBim Xplorer, shown in figure 8.

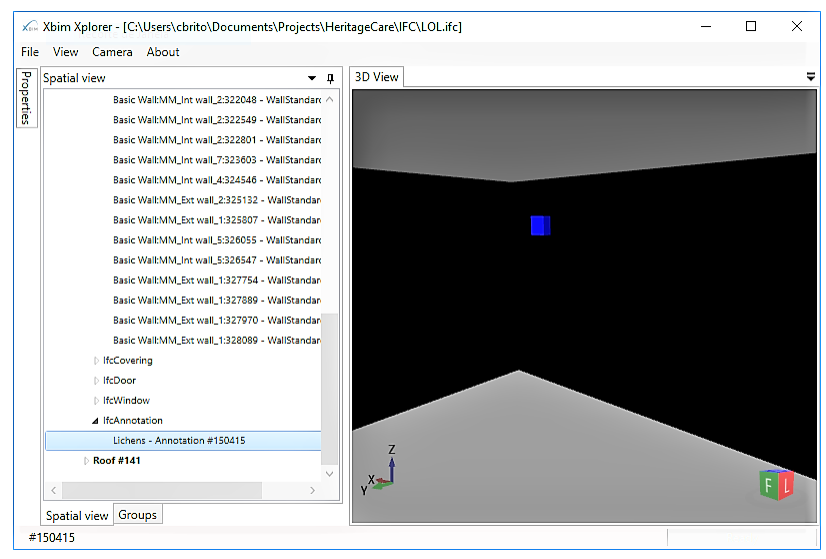

Figure 8. The Lichens annotation visualized in xBim Xplorer. 


\section{CONCLUSIONS}

In this article, we have presented the highlights of our Mixed Reality solution for heritage building inspection assistance. We found this to be an adequate use case for mixed reality and further demonstrates the vast applicability of this recent paradigm. The main difficulties we experienced are related to the device itself, and its intrinsic limitations, particularly regarding the automatic registration. This could be overcome with either enhanced hardware, or specifically designed algorithms. We also conclude that user interfaces for specific use cases such as this must be finely tailored so as to provide a non-obtrusive and efficient interaction.

\section{ACKNOWLEDGEMENTS}

This article is a result of the project HeritageCARE (SOE1/P5/P0258), cofunded by the European Regional Development Fund (ERDF) within the Interreg-SUDOE program. This work has been supported by FCT - Fundação para a Ciência e Tecnologia within the Project Scope: UID/CEC/00319/2019.

\section{REFERENCES}

Besl, P. J., McKay, N. D., 1992. Method for registration of 3-d shapes. Sensor Fusion IV: Control Paradigms and Data Structures, 1611, International Society for Optics and Photonics, 586-607.

Bouaziz, S., Tagliasacchi, A., Pauly, M., 2013. Sparse iterative closest point. Proceedings of the Eleventh Eurographics/ACMSIGGRAPH Symposium on Geometry Processing, Eurographics Association, 113-123.

Chi, H.-L., Kang, S.-C., Wang, X., 2013. Research trends and opportunities of augmented reality applications in architecture, engineering, and construction. Automation in construction, 33, $116-122$.

Fonnet, A., Alves, N., Sousa, N., Guevara, M., Magalhães, L., 2017. Heritage bim integration with mixed reality for building preventive maintenance. $201724^{\circ}$ Encontro Português de Computação Gráfica e Interação (EPCGI), IEEE, 1-7.

Gao, G., Liu, Y.-S., Wang, M., Gu, M., Yong, J.-H., 2015. A query expansion method for retrieving online BIM resources based on Industry Foundation Classes. Automation in construction, 56, 14-25.

Garon, M., Boulet, P.-O., Doironz, J.-P., Beaulieu, L., Lalonde, J.-F., 2016. Real-time high resolution $3 \mathrm{~d}$ data on the hololens. 2016 IEEE International Symposium on Mixed and Augmented Reality (ISMAR-Adjunct), IEEE, 189-191.

Gavish, N., Gutiérrez, T., Webel, S., Rodríguez, J., Peveri, M., Bockholt, U., Tecchia, F., 2015. Evaluating virtual reality and augmented reality training for industrial maintenance and assembly tasks. Interactive Learning Environments, 23(6), 778798.

Hanna, M. G., Ahmed, I., Nine, J., Prajapati, S., Pantanowitz, L., 2018. Augmented reality technology using microsoft hololens in anatomic pathology. Archives of pathology \& laboratory medicine, 142(5), 638-644.
Lucchi, E., 2018. Review of preventive conservation in museum buildings. Journal of Cultural Heritage, 29, 180-193.

Mesas-Carrascosa, F., Verdú Santano, D., Meroño de Larriva, J., Ortíz Cordero, R., Hidalgo Fernández, R., García-Ferrer, A., 2016. Monitoring heritage buildings with open source hardware sensors: A case study of the mosque-cathedral of Córdoba. Sensors, 16(10), 1620 .

Murphy, M., McGovern, E., Pavia, S., 2009. Historic building information modelling (HBIM). Structural Survey, 27(4), 311 327.

Perles, A., Perez-Marin, E., Mercado, R., Segrelles, J. D., Blanquer, I., Zarzo, M., Garcia-Diego, F. J., 2018. An energyefficient internet of things (IoT) architecture for preventive conservation of cultural heritage. Future Generation Computer Systems, 81, 566-581.

Rusinkiewicz, S., Levoy, M., 2001. Efficient variants of the icp algorithm. 3dim, IEEE, 145.

Succar, B., 2009. Building information modelling framework: A research and delivery foundation for industry stakeholders. Automation in construction, 18(3), 357-375.

Terminology, I., Resolution, C., 2008. Terminology to characterize the conservation of tangible cultural heritage. ICOM CC.

Wu, H.-K., Lee, S. W.-Y., Chang, H.-Y., Liang, J.-C., 2013. Current status, opportunities and challenges of augmented reality in education. Computers \& education, 62, 41-49. 